\title{
Plastic Modification of Anti-Saccades: Adaptation of Saccadic Eye Movements Aimed at a Virtual Target
}

\author{
Delphine Lévy-Bencheton, ${ }^{1,2}$ Laure Pisella, ${ }^{1,2}$ Roméo Salemme, ${ }^{1,2}$ Caroline Tilikete, ${ }^{1,2,3}$ and Denis Pélisson ${ }^{1,2}$ \\ ${ }^{1}$ Lyon Neuroscience Research Center, INSERM, Unit 1028, CNRS Unit 5292, ImpAct Team, F-69676 Bron, France, ${ }^{2}$ Lyon I University, F-69373 Lyon, France, \\ and ${ }^{3}$ Hospices Civil de Lyon, Neuro-Ophthalmology Unit, Neurological Hospital, F-69677 Bron, France
}

Saccades allow us to visually explore our environment. Like other goal-directed movements, their accuracy is permanently controlled by adaptation mechanisms that, in the laboratory, can be induced by systematic displacement of the "real" visual target during the saccade. However, in an anti-saccade (AS) task, the target is "virtual" because gaze has to be shifted away from the "real" visual target toward its mentally defined mirror position. Here, we investigated whether the brain can adapt movements aimed at a virtual target by trying, for the first time, to adapt AS. Healthy human volunteers produced leftward AS during three different exposure phases in which a visual target provided feedback after the AS. In the adaptation condition, the feedback target appeared after completion of the AS response at a location shifted outward from final eye position (immediate non-veridical feedback). In the two control conditions, adaptation was prevented by delaying $(800 \mathrm{~ms})$ the shifted feedback target (delayed-shift) or by providing an immediate but veridical feedback at the mirror position of the visual target (no-shift). Results revealed a significant increase of AS gain only in the adaptation condition. Moreover, testing pro-saccades (PS) before and after exposure revealed a significant increase of leftward PS gain in the adaptation condition. This transfer of adaptation supports the hypotheses of a motor level of AS adaptation and of a visual level of AS vector inversion. Together with data from the literature, these results also provide new insights into adaptation and planning mechanisms for AS and for other subtypes of voluntary saccades.

\section{Introduction}

Saccadic adaptation is a sensorimotor plasticity mechanism that permanently maintains saccade accuracy despite lasting physiological or pathological changes of the saccadic system. Adaptation of visually guided saccades is thus conceived as part of the foveation mechanism. Therefore, it might appear impossible to adapt anti-saccades (AS) because, in this task, the foveating prosaccade (PS) is inhibited and replaced by a voluntary saccade aimed at a "virtual" target located opposite to the "real" target (Hallett, 1978). Demonstrating plastic modification of AS would reveal that accuracy-keeping mechanisms of saccades are active even when they aim at a virtual target. If so, a transfer of AS adaptation to PS would further reveal whether common sensorimotor mechanisms between AS and foveating saccades take place at the visual or motor level.

When we produce a saccadic eye movement toward an object of interest, target eccentricity and movement extent match, defining similar vectors. In the AS task, these visual and motor

\footnotetext{
Received Jan. 8, 2013; revised July 5, 2013; accepted July 14, 2013.

Author contributions: D.L.-B., L.P., C.T., and D.P. designed research;D.L.-B. performed research; R.S. contributed unpublished reagents/analytic tools; D.L.-B. analyzed data; D.L.-B., L.P., C.T., and D.P. wrote the paper.

This work was funded by PHRC 2010 (C.T., principal investigator). This work was performed within the framework of the LABEX CORTEX (Grant ANR-11-LABX-0042) of University of Lyon, within the Investments in the Future Programme (Grant ANR-11-IDEX-0007) operated by the French National Research Agency. We thank M. Panouillères, A. Z. Khan, and P. Vindras for their comments on a previous version of this manuscript.

The authors declare no competing financial interests.

Correspondence should be addressed to Delphine Lévy-Bencheton, Lyon Neuroscience Research Center, INSERM, Unit 1028, CNRS Unit 5292, ImpAct Team, F-69676 Bron, France. E-mail: delphine.levy-bencheton@inserm.fr.

DOI:10.1523/JNEUROSCI.0077-13.2013

Copyright $\odot 2013$ the authors $\quad 0270-6474 / 13 / 3313489-09 \$ 15.00 / 0$
}

vectors are dissociated, because they point in opposite directions. Although neurophysiological studies could not conclude the visual or motor level of vector inversion during AS programming (Schlag-Rey et al., 1997; Sato and Schall, 2003; Schall, 2004; Zhang and Barash, 2004), Collins et al. (2008) demonstrated that vector inversion occurred upstream of the site of PS adaptation and concluded that it is the visual vector that is inverted. However, the level of PS adaptation is also debated because it may occur at the level of the interpretation of visual eccentricity or at the level of transforming the resulting visual vector into a motor vector. Different adaptation levels have been proposed depending on the category of PS and on the direction of the target step used in the McLaughlin (1967) adaptation protocol. Investigating the transfer of adaptation to other categories of saccades (including AS), hand movements, or perceptual localization judgments, several studies led to the general conclusion that reactive saccades (triggered by external stimuli) may adapt at a motor level, whereas voluntary saccades (triggered based on internal goals) may adapt at the visual level (Zimmermann et al., 2011; Zimmermann, 2013) or both visual and motor levels (Cotti et al., 2007, 2009; Zimmermann and Lappe, 2009; for review, see Pélisson et al., 2010). In parallel, it has been proposed that outward adaptation (increased amplitude) relies on earlier visual levels compared with inward adaptation (decreased amplitude) (Panouillères et al., 2009; Schnier et al., 2010; Zimmermann and Lappe, 2010; Zimmermann et al., 2011; Zimmermann, 2013). Here, we applied an outward adaptation protocol to voluntary leftward saccades elicited by right-sided visual targets (AS) and therefore expected adaptation of AS, if present, to occur at a 


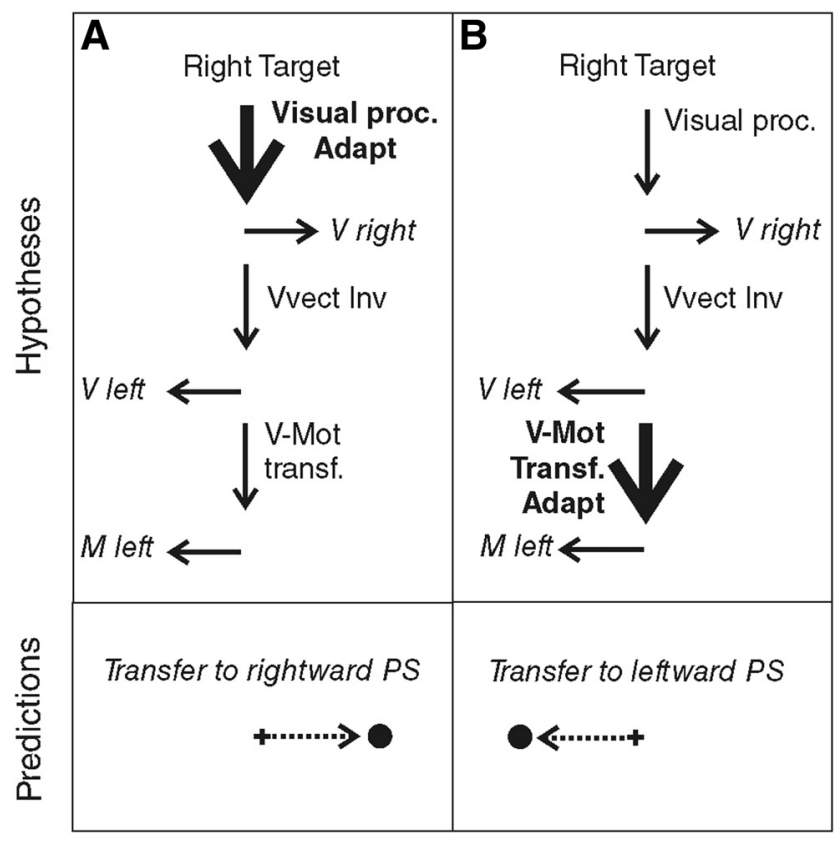

Figure 1. Predictions of the adaptation level of AS in case of visual vector inversion (VVect Inv). The schematics shown in the top indicate the hypothesized sites of adaptation (Adapt: bold font and thick arrow) of leftward AS. Visual proc., Visual processing; V-Mot transf., visuomotor transformation. The bottom represents the predicted transfer to PS. A, Visual adaptation hypothesis. Adaptation occurs at the level of visual processing of the target and leads to a transfer to rightward PS. B, Motor adaptation hypothesis. Adaptation occurs at a motor level, downstream of the vector inversion. In this case, adaptation of leftward AS should transfer to leftward PS.

visual level. According to this visual adaptation hypothesis (Fig. $1 A$ ), leftward AS adaptation should transfer to the rightward PS (with the same visual processing). In contrast, if we consider the motor adaptation hypothesis (Fig. $1 B$ ), we expect adaptation of leftward AS to transfer to the leftward PS (with the same motor vector), as long as it is the visual vector that is inverted (Collins et al., 2008).

\section{Materials and Methods}

Participants

Eight volunteers (six females, two males; age range, 23-35 years), without any neurological or ophthalmological history and with normal or corrected-to-normal vision, participated in the study. Each subject performed the three conditions described below, in three sessions separated by at least 1 week. All procedures were approved by the local ethical committee on human experimentation (Comités de Protection des Personnes Sud-Est III), in agreement with French law (March 4, 2002) and the Declaration of Helsinki (number 2008-057B). Written informed consent was obtained from all subjects.

\section{Experimental setup}

Subjects were seated $57 \mathrm{~cm}$ from a computer screen $(140 \mathrm{~Hz}$ vertical refresh rate), with their head maintained in a chin rest. They were presented visual targets $(0.6 \mathrm{~cm}$ black disks) on a gray background. The screen was controlled by a Visual Stimuli Generation system (Cambridge Research Systems). Horizontal and vertical positions of the right eye were continuously recorded using an infrared Eye-Tracker system (Cambridge Research Systems). An infrared camera was mounted above the chin rest, allowing high-frequency $(250 \mathrm{~Hz})$ acquisition of the eye images that were reflected by a $45^{\circ}$ tilted mirror located in front of the subject; the half-reflecting mirror allowed unrestricted, binocular vision. Before each session, eye-tracker signal was calibrated by asking the subject to successively fixate nine positions on the screen, providing right eye position with a resolution of $0.05^{\circ}$ and an accuracy of $0.125^{\circ}-0.25^{\circ}$.

\section{Adaptation and control exposure phases}

In the adaptation condition and the control conditions (delayed-shift and no-shift), subjects were submitted to an exposure phase during which they performed leftward AS and were provided with a movementcontingent feedback target.

Tasks common to all three exposure phases. Each subject looked at the central fixation point (FP). After a random time (range, 1100-1500 ms), a peripheral target was presented on the horizontal meridian, randomly at $6^{\circ}, 9^{\circ}$, or $12^{\circ}$ to the right of the central FP (overlap paradigm). The subject was instructed to maintain his/her eyes on the FP until the appearance of the peripheral target and then to execute a saccade of the same amplitude in the opposite direction (leftward) in a self-paced manner, as accurately as possible. The subject was required to execute this AS within $1 \mathrm{~s}$ after target presentation. As soon as the AS was detected (eyes reaching a velocity threshold of $80 \%$ s), the $\mathrm{FP}$ and peripheral target were extinguished. After completion of the AS (eye velocity below $30 \%$ ), a feedback target was presented for $500 \mathrm{~ms}$ at a location and a timing that depended on the condition (see below). Then, the feedback target disappeared and the subject had to shift his/her gaze back to the center of the screen in preparation of the next trial. A total of 240 trials ( 80 for each target position) were performed during the exposure phase of each condition, lasting $\sim 20 \mathrm{~min}$.

Spatiotemporal criterion used for feedback target presentation. In all exposure trials, the feedback target was presented only if the eye displacement met the following spatiotemporal criterion: the feedback target was presented as soon as the eye displacement reached a minimum of $90 \%$ of the target distance (spatial threshold), if this occurred in a time range of $500 \mathrm{~ms}$ after the initiation of ocular displacement (temporal window). This temporal window was used to include the execution of secondary saccades that are frequently observed in the AS task $(22 \%$ of the trials across all conditions). An example is illustrated in Figure 2 for a target presented at $9^{\circ}$ to the right: in this case, the threshold eye position was situated at $8.1^{\circ}$ to the left ( $90 \%$ of the desired saccade size). If the spatiotemporal criterion was fulfilled, after completion of either the primary AS (Fig. 2A) or additional corrective saccades (Fig. 2B), the feedback target was presented at a time and position described below, together with a short and high-pitch "success" sound $(450 \mathrm{~Hz}, 110 \mathrm{~ms})$. In $\sim 6 \%$ of the trials (across all three conditions), this spatial threshold was not reached by eye position within the allocated $500 \mathrm{~ms}$ time range (Fig. 2C); in this case, a longer and lower-pitch "error" sound ( $35 \mathrm{~Hz}, 931 \mathrm{~ms})$ occurred and the feedback target was not presented.

Adaptation condition. To establish whether it is possible to adapt AS, this condition was systematically performed first for each subject (Fig. $3 B 1$ ). To induce adaptation, the feedback target was systematically presented with a leftward shift (outward adaptation) representing $10 \%$ of the actual eye displacement with respect to the endpoint of eye movement detected online. The feedback target was turned on as soon as the spatiotemporal criterion was fulfilled, without any delay $(0 \mathrm{~ms})$.

Control conditions. The two control conditions were performed in random order and designed to induce the same AS responses as in the adaptation condition but without producing adaptation (Fig. 3B2,B3). The first condition (delayed-shift) was based on the fact that delaying the presentation of the feedback target is known to prevent adaptation ( $\mathrm{Fu}-$ jita et al., 2002), and the second condition (no-shift) used veridical feedback target information (no target shift). Each of the two control tasks differed from the adaptation condition by a single parameter. (1) For the delayed-shift condition (Fig. 3B2), the feedback target was presented at the same spatial location as in the adaptation condition ( $10 \%$ outward shift) but not immediately. Instead, the presentation of the feedback target was delayed by $800 \mathrm{~ms}$ from the time the spatiotemporal criterion was fulfilled. (2) In the no-shift condition (Fig. 3B3), the feedback target was presented at the same time as in the adaptation condition $(0 \mathrm{~ms}$ delay) but at the true mirror target position, regardless of the final eye position.

\section{Pre-phase and post-phase}

PS and AS without feedback were recorded immediately before (pre-) and after (post-) the exposure phase for each adaptation or control condition. The FP location and duration, the saccade detection threshold, 

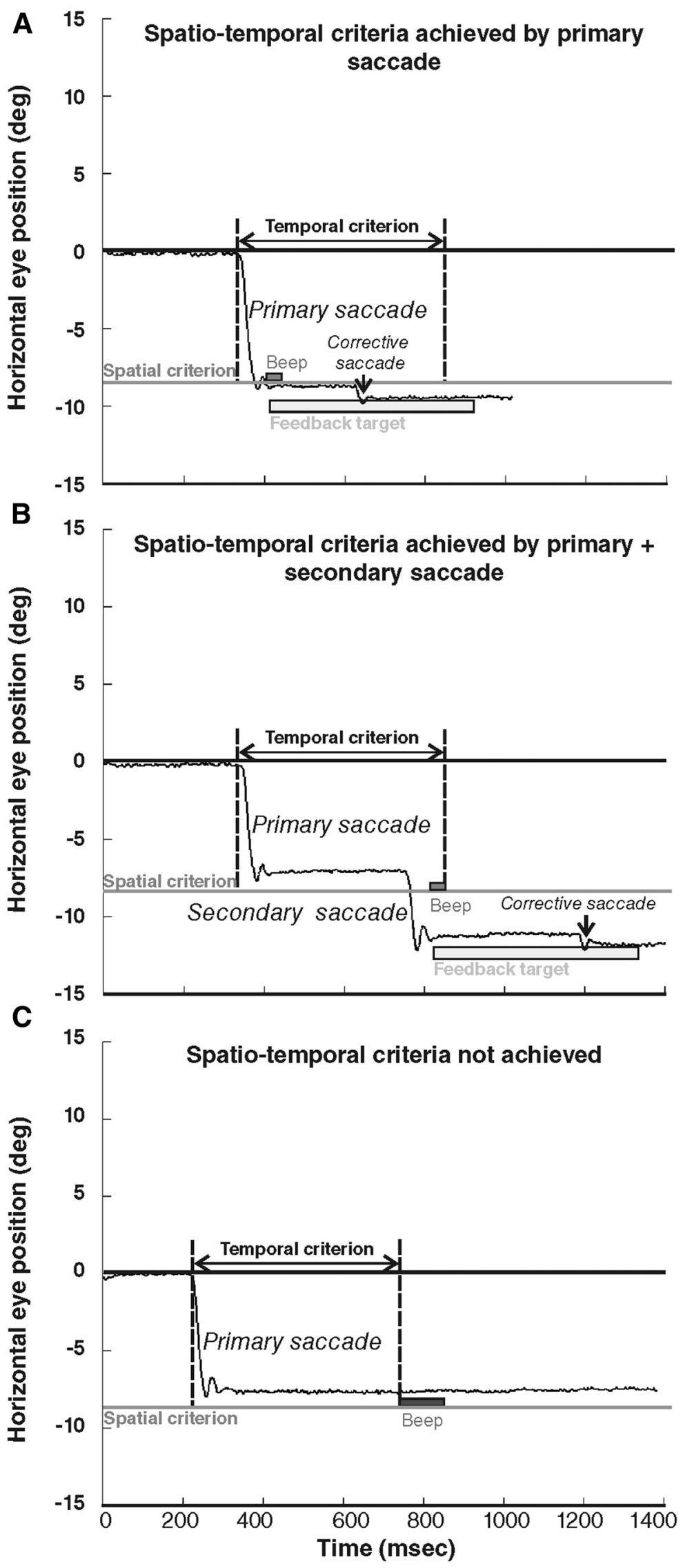

Figure 2. Oculomotor behavior during the adaptation exposure phase, exemplified by responses of subject 2 to the $9^{\circ}$ target The black line represents the horizontal eye position (in degrees) according to time (in milliseconds). Negative values represent left eye positions. The gray line represents the minimum amplitude required for the feedback target occurrence (spatial criterion). The and the sound signaling the end of each trial were the same as during the exposure phases. Other parameters differed as detailed below.

PS. Simultaneously with the extinction of the central FP, a peripheral target was presented randomly at $9^{\circ}$ in the right or left visual field, on the horizontal meridian. The subject was asked to look toward this peripheral target, which was extinguished as soon as saccade onset was detected. There were a total of 24 trials (12 trials for each direction) before and after exposure in each condition.

$A S$. While the FP was still on the screen, a peripheral target was presented randomly at $9^{\circ}$ in the right or left visual field, on the horizontal meridian (Fig. 3A). The subject had to produce a saccade in the direction opposite to this peripheral target with an amplitude corresponding to the target eccentricity. FP and peripheral target were simultaneously switched off as soon as saccade onset was detected. There were a total of 24 trials ( 12 for each direction) before and after exposure in each condition.

In the pre-phase, PS were tested first, whereas in the post-phase, AS were tested first. Indeed, classically, the adapted saccades (here AS) are recorded immediately before and after the adaptation procedure (pre-phase and postphase, respectively) with no visual feedback to test for adaptation aftereffects (retention). Consequently, saccades of another category (here PS) are elicited at the beginning and the end of the pre-phase and post-phase, also with no visual feedback, to test for adaptation transfer.

\section{Data analyses}

Horizontal eye movements were analyzed offline using a program developed in the laboratory under MATLAB version 7.8 (MathWorks). The beginning and end of saccades were automatically detected on the basis of $80 \%$ s velocity threshold. They were verified and corrected when necessary by the experimenter. The actual saccade amplitude was calculated as the difference between the final and initial eye positions. The desired saccade amplitude was calculated as the difference between the peripheral target position and the initial eye position. The gain of each primary saccade was calculated as the ratio between the actual and desired saccade amplitudes.

\footnotetext{
$\leftarrow$

temporal criterion is the $500 \mathrm{~ms}$ period initiated at the beginning of the saccade (vertical dotted lines). In $\boldsymbol{A}$ and $\boldsymbol{B}$, the eyes crossed the spatial criterion by one $(\boldsymbol{A})$ or two $(\boldsymbol{B})$ saccades executed in due time relative to the temporal criterion. Light gray rectangle illustrates the $500 \mathrm{~ms}$ presentation of the feedback target occurring at the completion of the first saccade that crossed the spatial criterion (note the corrective saccade elicited by the feedback target). In C, the primary saccade does not reach the spatial criterion, and no other saccade was performed by the subject within the temporal criterion; therefore, no feedback target was presented. Auditory feedback is also represented immediately after the saccade crossed the spatial criterion in $\boldsymbol{A}$ and $\boldsymbol{B}$ (high-pitch "success" sound) and at the end of the temporal criterion in C (low-pitch "error" sound).
} 
Anti-saccade task during pre- / post-phase and exposure phase

\section{A Pre/Post-phase B Exposure phase}



Figure 3. Anti-saccade task during pre-phase, post-phase, and exposure phase. In all cases (pre/post-phase and exposure phase), the FP is presented first, followed by a peripheral target presented in the right visual field (at $9^{\circ}$ in these examples). The AS (thick black line) is performed in a self-paced manner to the left visual field (downward deflection). Both $\mathrm{FP}$ and target are extinguished at AS onset. In the pre-phase and post-phase $(\boldsymbol{A})$, no feedback target is provided, whereas in exposure phase $(\boldsymbol{B})$, a feedback target is systematically presented (FT, light gray rectangle), with different parameters according to the condition. In the adaptation condition (B1), the feedback target is presented at the time of AS completion ( $0 \mathrm{~ms})$, at a location shifted outward from the AS response endpoint by a shift representing $10 \%$ of the AS response amplitude. In the delayed-shift condition (B2), the feedback target is also presented at the shifted location (same $10 \%$ shift) but after a $800 \mathrm{~ms}$ delay. In the no-shift condition (B3), the feedback target is presented immediately after AS completion ( $0 \mathrm{~ms}$ delay) but at the mirror position of the peripheral target (no shift). Note that, in the pre-phase and post-phase, both rightward AS and rightward and leftward PS were also performed (data not shown).

Exposure phase analyses. For each subject, the regression slope of the relationship between primary saccade gain and time (trial number) was calculated, separately for the three target eccentricities and the three conditions. Subjects had been encouraged to blink only after the end of each trial, when moving their eyes back to the center of the screen, such that only a few trials with blinks had to be removed $(1.63 \pm 0.26 \%$ of total trials).

Pre-phase and post-phase analyses. For each subject, the mean saccadic gain was calculated separately for PS and AS, for left and right targets, and for the pre-phase and post-phase. Trials with blink were removed (1.51 \pm $0.58 \%$ of total trials). To evaluate the adaptation aftereffect of AS and the transfer of adaptation to PS, we calculated the percentage gain change between pre-phase and post-phase as follows:

Percent gain change $=\frac{\text { mean gain post }- \text { mean gain pre }}{\text { mean gain pre }} \times 100$.

We noticed that, despite the fact that the target was extinguished at saccade onset in pre-phase and post-phase, secondary saccades were often (30\% of the trials) generated after the primary saccade execution, in either the same (outward) or the opposite (inward) direction as the primary saccade. Thus, we performed an additional analysis of percentage gain change between pre-phase and post-phase in which the total eye displacement was computed by taking into account all secondary saccades that occurred within $500 \mathrm{~ms}$ after the offset of primary saccade.

\section{Statistical analyses}

Statistical analyses were performed with the Statistica 9 software (Statsoft). For the exposure phase, the regression slope of the saccade gain versus trial number relationship was computed for each subject and each target eccentricity. A factorial ANOVA was performed to test the potential effect of condition and eccentricity on the regression slopes. If a condition effect was demonstrated, the regression slopes were compared with a $t$ test to the standard value $(0)$ for each condition to decide whether saccade gain evolved significantly over the time of exposure. For the pre-phase and post-phase, three-way ANOVAs were performed on the gain of both the primary saccade and the total eye displacement, separately for each condition, with type (AS, PS), direction (leftward, rightward), and phase (pre-, post-) as factors. If a phase effect was demonstrated, the percentage gain changes were compared with a $t$ test to the standard value (0), for both the primary saccade and for the total eye displacement, to assess any significant gain change between pre-phase and post-phase. Significant level was set at $p<0.05$ and corrected for multiple comparisons with false discovery rate (FDR; Benjamini and Hochberg, 1995).

\section{Results}

To assess the efficiency of adaptation, we analyzed the changes of AS gain that took place both during the exposure phase and the post-phase relative to the pre-phase.

\section{AS gain changes during exposure phases}

Example of subject 4

In the adaptation condition, the regression slopes of the relationship between AS gain and trial number for the $6^{\circ}, 9^{\circ}$, and $12^{\circ}$ target eccentricities were positive, i.e., signaling an increase of AS gain, and significantly different from 0 (target regression slope $=$ $0.0014, p=0.0092$; target regression slope $=0.0013, p=0.0007$; and target regression slope $=0.001, p<0.0001$, respectively; Fig. $4 A)$. For the control conditions, only the no-shift condition revealed a significant gain decrease of AS to the $12^{\circ}$ target (slope $=$ $-0.0005, p=0.0039$; Fig. $4 C)$.

\section{All subjects}

A factorial ANOVA was performed on the regression slopes of all subjects to test the effects of target eccentricity and conditions (Fig. $4 D)$. There was no effect of eccentricity $\left(F_{(2,42)}=1.60, p=\right.$ $0.225)$ and also no interaction between condition and eccentricity $\left(F_{(4,42)}=0.134, p=0.96\right)$ but a main effect of condition $\left(F_{(2,4)}=4.51, p=0.016\right)$. We observed a significant gain increase during the exposure phase in the adaptation condition (regression slope significantly different from $0: t_{(23)}=2.58, p=0.0165$ ) but not in the control conditions (delayed-shift, $t_{(23)}=0.46, p=$ 0.64 ; no-shift, $t_{(23)}=-0.91, p=0.37$; FDR-corrected $p$ value for multiple comparisons $=0.0166)$.

\section{Adaptation aftereffects and transfers}

Latencies of AS (adaptation, $314 \mathrm{~ms}$; delayed-shift, $331 \mathrm{~ms}$; noshift, $314 \mathrm{~ms}$ ) were longer than latencies of PS (adaptation, 215 

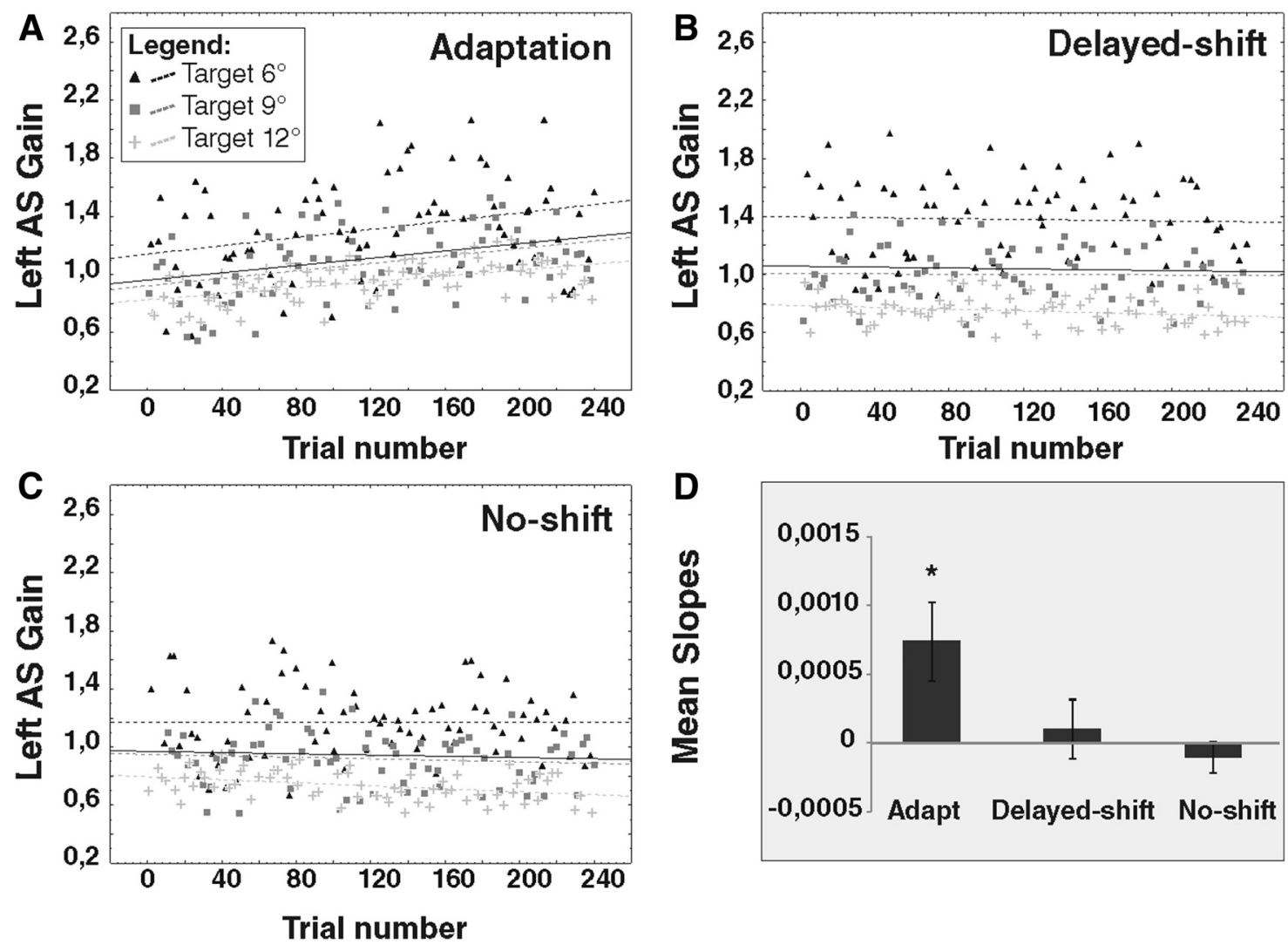

Figure 4. Regression slopes ofleft AS gain for one representative subject (subject 4) during exposure phases. The gain of leftward AS (L-AS) performed during the exposure phase of the adaptation condition $(\boldsymbol{A})$, delayed-shift condition $(\boldsymbol{B})$, and no-shift condition $(\boldsymbol{C})$ is represented separately for each target $\left(6^{\circ}\right.$, black triangles; $9^{\circ}$, dark gray squares; $12^{\circ}$, light gray plus signs). Dotted lines represent the slopes of the linear relationship between AS gain and trial number for each target. Black thick line represents the slope of the averaged relationship of the three target eccentricities pooled together. In the adaptation condition, the slope of all three relationships $\left(6^{\circ}, 9^{\circ}, 12^{\circ}\right)$ between AS gain and trial number is significantly larger than 0 . In the no-shift condition, the slope of the $12^{\circ}$ relationship is significantly smaller than 0 . All other relationships are not significant. D represents the slopes averaged over all subjects separately for each condition. Asterisk indicates significant differences relative to 0 ( $t$ tests).

ms; delayed-shift, $226 \mathrm{~ms}$; no-shift, $227 \mathrm{~ms}$ ), as classically observed in the literature (Hallett, 1978; Munoz and Everling, 2004).

Repeated-measures ANOVA were performed on the primary saccade gain and on the total eye displacement gain (including secondary saccades made within $500 \mathrm{~ms}$ after the offset of primary saccade) with the following four factors: (1) condition (adaptation, delayed-shift, no-shift); (2) type (AS, PS); (3) direction (leftward, rightward); and (4) phase (pre-, post-). With both gain parameters, we found a main effect of condition $\left(F_{(2,14)}=6.536\right.$, $\left.p=0.009 ; F_{(2,14)}=5.462, p=0.017\right)$, which led us to perform three-way repeated-measures ANOVAs for each condition separately.

\section{Adaptation condition}

The three-way repeated-measures ANOVA revealed a direction $X$ phase interaction that just reached significance for the primary saccade gain $\left(F_{(1,7)}=5.486, p=0.05\right)$ and that was also significant for the total eye displacement gain $\left(F_{(1,7)}=10.89, p=\right.$ 0.013 ; Fig. 5 , left column). There was no significant type $\times$ phase interaction $\left(F_{(1,7)}=2.34, p=0.169\right.$ for primary; $F_{(1,7)}=3.30$, $p=0.11$ for total eye displacement) nor any three-level interaction $\left(F_{(1,7)}=0.145, p=0.71 ; F_{(1,7)}=2.85, p=0.13\right.$ for primary and total eye displacement, respectively). These results indicate that pre-post differences of saccade gain related to AS adaptation depend only on the motor direction of saccades and not on their type (AS or PS). Indeed, the percentage gain change of rightward
AS and rightward PS did not differ from 0 for both primary saccade $\left(t_{(7)}=0.63, p=0.54 ; t_{(7)}=-1.10, p=0.30\right.$, respectively) and total eye displacement analyses $\left(t_{(7)}=-0.17, p=0.86 ; t_{(7)}=\right.$ $-1.12, p=0.29$, respectively). In contrast, the percentage gain change of primary saccades for the leftward AS (10.8\%) and leftward PS $(3.2 \%)$ differed significantly from $0\left(t_{(7)}=2.49, p=\right.$ $0.041 ; t_{(7)}=3.36, p=0.01$, respectively; Fig. 5 , left column) but remained significant only for the leftward PS after correction for multiple comparisons (FDR-corrected $p$ value $=0.025$ ). Moreover, when we took into account the corrective saccade in the total eye displacement analysis, the gain increase of the leftward AS (12.8\%) and the leftward PS (2.9\%) still significantly differed from $0\left(t_{(7)}=3.43, p=0.0109 ; t_{(7)}=3.30, p=0.0129\right.$, respectively; FDR-corrected $p$ value for multiple comparisons $=0.025$ ). In contrast, for rightward saccades, the percentage gain change remained nonsignificant in the total eye displacement analysis $t_{(7)}=-0.17, p=0.86 ; t_{(7)}=-1.12, p=0.29$, respectively, for rightward AS and rightward PS), confirming the specificity of adaptation transfer to leftward PS. The percentage of transfer (percentage gain change of leftward PS divided by percentage gain change of leftward AS) comes up to $18.8 \%$ in the primary saccade analysis and to $14.6 \%$ in the total eye displacement analysis.

\section{Delayed-shift condition}

The three-way repeated-measures ANOVA in the delayed-shift condition revealed significant effects of the type $\times$ direction $\times$ 


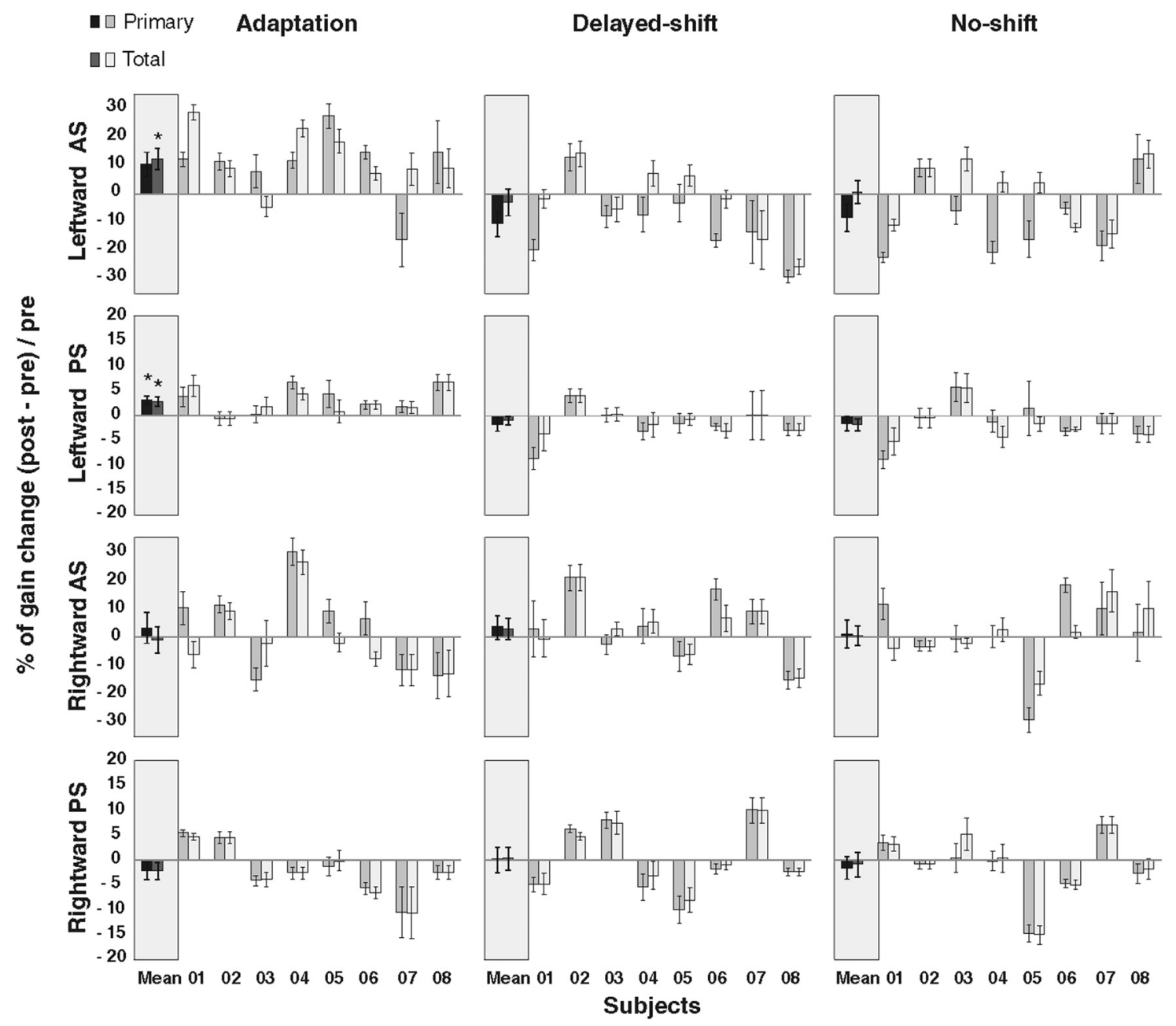

Figure 5. Percentage gain change of primary saccade and of total eye displacement during pre-phase and post-phase. Positive (negative) value refers to a gain increase (decrease) in post-phase compared with pre-phase. Each subject (1 to 8) is represented for each condition, direction, and type of saccades, as well as the average over all subjects (mean). The first (darker) bar of each pair represents the gain change of the primary saccade, whereas the second (lighter) bar is the gain change of the total eye displacement. Asterisks indicate significant differences relative to 0 ( $t$ tests). Error bars represent SE.

phase interaction and of the direction $\times$ phase interaction $\left(F_{(1,7)}=10.17, p=0.015 ; F_{(1,7)}=7.36, p=0.03\right.$, respectively) for the primary saccade gain and only a nonsignificant type $\times$ direction $\times$ phase interaction effect for the total eye displacement gain $\left(F_{(1,7)}=3.88, p=0.09\right.$; Fig. 5, middle column). The percentage gain change of leftward AS between the pre-phase and post-phase $(-10.3 \%$ in the primary analysis and $-2.5 \%$ in the total eye displacement analysis) did not differ from $0\left(t_{(7)}=-2.33, p=\right.$ $0.052 ; t_{(7)}=-0.55, p=0.59$, respectively, for the two analyses), as well as for the other type and direction of saccades (for primary saccades and total eye displacement, respectively: rightward AS, $t_{(7)}=0.85, p=0.42 ; t_{(7)}=0.82, p=0.43$; leftward PS, $t_{(7)}=$ $-1.35, p=0.22 ; t_{(7)}=-1.02, p=0.34$; rightward PS, $t_{(7)}=0.02$, $\left.p=0.98 ; t_{(7)}=0.18, p=0.85\right)$.

\section{No-shift condition}

The three-way repeated-measures ANOVA did not reveal any significant effect in the no-shift condition in both the primary saccades and the total eye displacement analyses (Fig. 5, right column). Accordingly, the percentage gain change between the pre-phase and post-phase for leftward AS $(-8.02 \%$ with primary saccade analysis and $1.1 \%$ for total eye displacement analysis) was not different from $0\left(t_{(7)}=-1.69, p=0.13 ; t_{(7)}=0.27, p=0.79\right.$, respectively, for the two analyses), as well as for the other types and directions of saccades (for primary saccades and total eye displacement, respectively: rightward AS, $t_{(7)}=0.23, p=0.82$; $t_{(7)}=0.18, p=0.85$; leftward PS, $t_{(7)}=-0.96, p=0.37 ; t_{(7)}=$ $-1.44, p=0.19$; rightward PS, $t_{(7)}=-0.65, p=0.53 ; t_{(7)}=$ $-0.32, p=0.75)$.

Number and direction of secondary saccades in pre-phase and post-phase

To understand the differences between the primary saccade and the total eye displacement analyses observed for leftward AS (Fig. 5 ), we calculated the number of inward and outward secondary saccades in pre-phase and post-phase (Table 1).

$\chi^{2}$ tests allowed us to test for each condition (adaptation, delayed-shift, and no-shift) whether the relative number of inward and outward secondary saccades significantly differed between pre-phase and post-phase. Results showed that there were many more outward secondary saccades in addition to a decrease of inward saccade number in the post-phase compared with the pre-phase for the delayed-shift and the no-shift conditions $\left(\chi^{2}=\right.$ $10.99, p<0.001 ; \chi^{2}=14.83, p<0.001$, respectively). These changes compensated for the gain decrease of leftward primary AS observed during the control exposure phases. In the adap- 
Table 1. Number and direction of secondary saccades in pre-phase and post-phase

\begin{tabular}{|c|c|c|c|c|c|c|}
\hline & \multicolumn{2}{|l|}{ Adapt } & \multicolumn{2}{|c|}{ Delayed-shift } & \multicolumn{2}{|l|}{ No-shift } \\
\hline & Pre-phase & Post-phase & Pre-phase & Post-phase & Pre-phase & Post-phase \\
\hline Inward & 15 & 15 & 14 & 3 & 13 & 2 \\
\hline Outward & 11 & 20 & 15 & 28 & 10 & 26 \\
\hline All & 26 & 35 & 29 & 31 & 23 & 28 \\
\hline
\end{tabular}

$\chi^{2}$ tests were performed to test the difference between pre-phase and post-phase of the relative number of inward and outward secondary saccades. Number of secondary saccades averaged over all subjects for the leftward AS trials, in the pre-phase and post-phase of the three conditions.

tation condition, no significant change was found between the relative number of inward and outward secondary saccades $\left(\chi^{2}=1.31, p=0.25\right)$.

\section{Discussion}

This study is the first demonstration of adaptation of AS. A significant increase of AS gain was observed during the exposure phase, only in the adaptation condition. In addition, this adaptive effect persisted after the adaptation exposure phase, resulting in a gain increase in the post-phase compared with the pre-phase (aftereffect), reaching significance when secondary saccades were taken into account. Srimal and Curtis (2010) have already reported a contribution of secondary saccades to the adaptation of memory-guided saccades, a task in which saccades are made to the remembered location of a previously flashed target and thus, like in the AS task, to a mental representation of the target, i.e., a "virtual" target. Despite there being no visual feedback during the pre-phase and post-phase, eye displacement sometimes continued after the primary saccade execution, as if the subject felt that he/she was inaccurate with respect to his mental representation of target position. This interpretation of a role of secondary saccades is also highly consistent with the control conditions in which the (nonsignificant) decrease of primary saccades gain between the pre-phase and post-phase, probably as a result of fatigue (Golla et al., 2008), was compensated for by an increase of the number of outward secondary saccades, thus leading to a very small residual gain change of total eye displacement.

In our paradigm, we provided different visual and auditory feedbacks according to the correct or incorrect execution of AS (i.e., matching or not the spatiotemporal criterion). Delivering such types of sensory feedback contingent on saccade performance has been shown to produce saccade amplitude changes through reinforcement learning (Madelain et al., 2011). However, given that in our study the spatiotemporal criterion to provide these feedbacks was exactly the same in all three conditions, we are confident that the AS gain change demonstrated specifically in the adaptation condition results from sensorimotor adaptation mechanisms. This conclusion is further supported by the fact that this change matches some well-known characteristics of adaptation: (1) the gain of rightward AS was never modified in any of the three conditions (directional specificity); and (2) the gain change persisted in the post-adaptation phase (aftereffect).

In summary, this study demonstrates that accuracykeeping mechanisms known so far for saccades aimed at a visual target are active even when saccades are aimed at a virtual target. This similarity suggests common visuomotor control mechanisms between foveating and non-foveating saccades, but which visual or motor level will be discussed in the following paragraphs.

We could expect that outward adaptation of AS would involve an early level of the visuomotor chain of processing

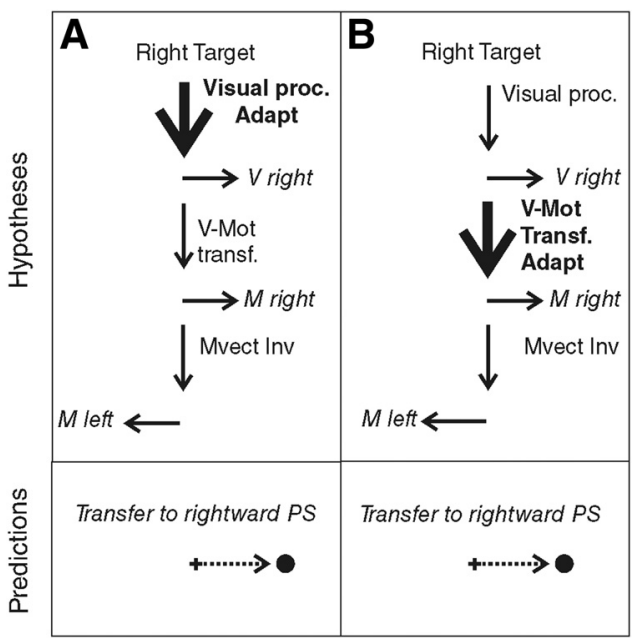

Figure 6. Predictions of the adaptation level of AS in case of motor vector inversion (Mvect Inv.). As in Figure 1, the top indicates the hypothesized sites of adaptation (Adapt: bold font and thick arrow) of leftward AS, and the bottom represents the predicted transfer to PS. Visual proc., Visual processing; V-Mot transf., visuomotor transformation. $\boldsymbol{A}$, Visual adaptation hypothesis. Adaptation occurs at the level of visual processing of the target, upstream of the motor vector inversion (Mvect Inv.) and thus leads to a transfer to rightward PS. B, Motor adaptation hypothesis. Adaptation occurs at a motor level but still upstream of the motor vector inversion, thus again transferring to right-PS.

because (1) the outward adaptation procedure has been proposed to take place at the visual level (Panouillères et al., 2009; Schnier et al., 2010; Zimmermann and Lappe, 2010; Schnier and Lappe, 2011), and (2) the adaptation of scanning saccades, another subtype of voluntary saccades, has been suggested to involve a visual level (Cotti et al., 2007, 2009; Schnier et al., 2010; Zimmermann and Lappe, 2010; Zimmermann et al., 2011; Zimmermann, 2013). Accordingly, we predicted that the processing (Fig. 1, visual proc) leading to the right visual vector (Fig. 1, V right) would be modified during adaptation of leftward AS, which should then transfer to the rightward PS. Contrasting with this prediction, we did not observe any modification of rightward PS but instead a significant increase of the leftward PS gain, regardless of whether secondary saccades were taken into account. This transfer of leftward AS specifically to leftward PS (saccades of same motor direction) suggests that AS adaptation does not occur at a visual level but is rather confined at a motor level (i.e., a modification of the $M$ left vector in Fig. 1B).

Another main implication of this study is to address directly the level (visual or motor) at which both adaptation and vector inversion of AS programming take place. In contrast to previous studies investigating AS in relation to saccadic adaptation (Collins et al., 2008; Cotti et al., 2009; Panouillères et al., 2009), we could directly address each of these two questions without making any assumption on the other. Indeed, the transfer of AS adaptation to PS performed in the same (leftward) direction fits exclusively with the prediction that combines the motor adaptation hypothesis with the visual vector inversion hypothesis (Fig. $1 B$ ), with the latter being consistent with the conclusion of Collins et al. (2008). Had we found a significant transfer to rightward PS, no distinction could have been provided between the hypothesis illustrated in Figure $1 \mathrm{~A}$ and those associated with a motor vector inversion illustrated in Figure 6, $A$ (visual adaptation hypothesis) and $B$ (motor adaptation hypothesis). 
Finally, the percentage of adaptation transfer to PS allowed us to discuss the status of AS with respect to other subtypes of voluntary saccades and to speculate on the neural substrate of adaptation of saccadic responses toward virtual targets. The percentage of transfer to leftward PS (reactive saccades) was only partial $(18.8 \%)$, suggesting that, although at a purely motor level (no transfer to rightward PS; see above), the site of AS adaptation cannot be a neural substrate that is common for the execution of all types of saccades (such as the brainstem, cerebellum, or superior colliculus). Instead, this partial transfer indicates a partial overlapping between the neural substrates involved in the motor programming of AS (nonvisually guided voluntary saccades) and those involved in the motor programming of PS (visually guided reactive saccades). How does this percentage of transfer to reactive saccades of the same motor direction compare with the transfer of other types of voluntary saccades? Previous studies testing the transfer of voluntary saccades adaptation to reactive saccades showed that scanning saccades adaptation does transfer well (79\%) (Alahyane et al., 2007), whereas memory-guided saccades adaptation transfers much less (14.3\%) (Fujita et al., 2002). This suggests that the programming of reactive saccades (1) shares a common substrate near the final common pathway with scanning saccades but in contrast (2) only shares restricted substrate with memory-guided saccades. Because we also suggest here a partial overlapping between the neural substrates involved in AS and in reactive saccades programming, these transfer studies altogether suggest that AS might share neural substrates with memory-guided saccades. This last assumption is consistent with AS and memory-guided saccades both belonging to the non-visually guided category of voluntary saccades (Deubel, 1999; Hopp and Fuchs, 2010). Indeed, in both cases, visual information about goal location is absent at the time of saccade initiation. A parallel can be drawn between the possible neural substrates of these two types of nonvisually guided saccades, both involving the prefrontal cortex in their generation (Munoz and Everling, 2004). As for the site of adaptation of memory-guided saccades, a study showed deficits in Parkinson's disease patients, and the authors of this study suggested an involvement of the basal ganglia or of the output of the dorsolateral prefrontal cortex to the basal ganglia (MacAskill et al., 2002). Our interpretation regarding the level of AS vector inversion might be relevant regarding these possible cortical and subcortical substrates for AS adaptation. Indeed, our study suggests that AS adaptation is located downstream of the neural substrates of AS vector inversion. Neurophysiological studies have repeatedly demonstrated that the vector inversion could take place in the prefrontal cortex $(\mathrm{Fu}-$ nahashi et al., 1993), the frontal eye field (Sato and Schall, 2003; Schall, 2004; Moon et al., 2007), or parietal eye field (Zhang and Barash, 2000; Nyffeler et al., 2007; Van Der Werf et al., 2008). Then, assuming that, as discussed above, the AS adaptation site is similar to the memory-guided saccade adaptation site, our conclusion supports the hypothesis that AS adaptation also involves subcortical structures, such as the basal ganglia. Testing this hypothesis will require additional behavioral studies measuring directly the reciprocal transfers of adaptation.

In conclusion, the present study demonstrates that AS can be adapted, thereby proving that the brain can adapt movements aimed at virtual targets. In addition, the adaptation transfer to PS of the same motor direction allowed us to locate the site of AS adaptation relative to the site of AS vector inversion but also the adaptation site of AS relative to other types of voluntary saccades. Finally, in combination with previous studies, we suggest that memory-guided saccades and AS share common adaptive substrates.

\section{References}

Alahyane N, Salemme R, Urquizar C, Cotti J, Guillaume A, Vercher JL, Pélisson D (2007) Oculomotor plasticity: are mechanisms of adaptation for reactive and voluntary saccades separate? Brain Res 1135:107-121. CrossRef Medline

Benjamini Y, Hochberg Y (1995) Controlling the false discovery rate: a practical and powerful approach to multiple testing. J R Stat Soc Ser B 57:289-300.

Collins T, Vergilino-Perez D, Delisle L, Doré-Mazars K (2008) Visual versus motor vector inversions in the antisaccade task: a behavioral investigation with saccadic adaptation. J Neurophysiol 99:2708-2718. CrossRef Medline

Cotti J, Guillaume A, Alahyane N, Pelisson D, Vercher JL (2007) Adaptation of voluntary saccades, but not of reactive saccades, transfers to hand pointing movements. J Neurophysiol 98:602-612. CrossRef Medline

Cotti J, Panouilleres M, Munoz DP, Vercher JL, Pélisson D, Guillaume A (2009) Adaptation of reactive and voluntary saccades: different patterns of adaptation revealed in the antisaccade task. J Physiol 587:127138. CrossRef Medline

Deubel H (1999) Separate mechanisms for the adaptive control of reactive, volitional, and memory-guided saccadic eye movements. In: Attention and performance XVII cognitive regulation of performance: interaction of theory and application (Gopher D, Koriat A, eds), pp 697-721. Cambridge, MA: Massachusetts Institute of Technology.

Fujita M, Amagai A, Minakawa F, Aoki M (2002) Selective and delay adaptation of human saccades. Brain Res Cogn Brain Res 13:41-52. CrossRef Medline

Funahashi S, Chafee MV, Goldman-Rakic PS (1993) Prefrontal neuronal activity in rhesus monkeys performing a delayed anti-saccade task. Nature 365:753-756. CrossRef Medline

Golla H, Tziridis K, Haarmeier T, Catz N, Barash S, Thier P (2008) Reduced saccadic resilience and impaired saccadic adaptation due to cerebellar disease. Eur J Neurosci 27:132-144. CrossRef Medline

Hallett PE (1978) Primary and secondary saccades to goals defined by instructions. Vision Res 18:1279-1296. CrossRef Medline

Hopp JJ, Fuchs AF (2010) Identifying sites of saccade amplitude plasticity in humans: transfer of adaptation between different types of saccade. Exp Brain Res 202:129-145. CrossRef Medline

MacAskill MR, Anderson TJ, Jones RD (2002) Adaptive modification of saccade amplitude in Parkinson's disease. Brain 125:1570-1582. CrossRef Medline

Madelain L, Paeye C, Wallman J (2011) Modification of saccadic gain by reinforcement. J Neurophysiol 106:219-232. CrossRef Medline

McLaughlin S (1967) Parametric adjustment in saccadic eye movement. Percept Psychophys 2:359-362. CrossRef

Moon SY, Barton JJ, Mikulski S, Polli FE, Cain MS, Vangel M, Hämäläinen MS, Manoach DS (2007) Where left becomes right: a magnetoencephalographic study of sensorimotor transformation for antisaccades. Neuroimage 36:1313-1323. CrossRef Medline

Munoz DP, Everling S (2004) Look away: the anti-saccade task and the voluntary control of eye movement. Nat Rev Neurosci 5:218-228. CrossRef Medline

Nyffeler T, Müri RM, Bucher-Ottiger Y, Pierrot-Deseilligny C, Gaymard B, Rivaud-Pechoux S (2007) Inhibitory control of the human dorsolateral prefrontal cortex during the anti-saccade paradigm - a transcranial magnetic stimulation study. Eur J Neurosci 26:1381-1385. CrossRef Medline

Panouillères M, Weiss T, Urquizar C, Salemme R, Munoz DP, Pélisson D (2009) Behavioral evidence of separate adaptation mechanisms controlling saccade amplitude lengthening and shortening. J Neurophysiol 101: 1550-1559. CrossRef Medline

Pélisson D, Alahyane N, Panouillères M, Tilikete C (2010) Sensorimotor adaptation of saccadic eye movements. Neurosci Biobehav Rev 34:11031120. CrossRef Medline

Sato TR, Schall JD (2003) Effects of stimulus-response compatibility on neural selection in frontal eye field. Neuron 38:637-648. CrossRef Medline 
Schall JD (2004) On the role of frontal eye field in guiding attention and saccades. Vision Res 44:1453-1467. CrossRef Medline

Schlag-Rey M, Amador N, Sanchez H, Schlag J (1997) Antisaccade performance predicted by neuronal activity in the supplementary eye field. Nature 390:398-401. CrossRef Medline

Schnier F, Lappe M (2011) Differences in intersaccadic adaptation transfer between inward and outward adaptation. J Neurophysiol 106: 1399-1410. CrossRef Medline

Schnier F, Zimmermann E, Lappe M (2010) Adaptation and mislocalization fields for saccadic outward adaptation in humans. J Eye Mov Res 3:1-18.

Srimal R, Curtis CE (2010) Secondary adaptation of memory-guided saccades. Exp Brain Res 206:35-46. CrossRef Medline

Van Der Werf J, Jensen O, Fries P, Medendorp WP (2008) Gamma-band activity in human posterior parietal cortex encodes the motor goal during delayed prosaccades and antisaccades. J Neurosci 28:8397-8405. CrossRef Medline
Zhang M, Barash S (2000) Neuronal switching of sensorimotor transformations for antisaccades. Nature 408:971-975. CrossRef Medline

Zhang M, Barash S (2004) Persistent LIP activity in memory antisaccades: working memory for a sensorimotor transformation. J Neurophysiol 91: 1424-1441. Medline

Zimmermann E (2013) Reference frames in saccade adaptation. J Neurophysiol 109:1815-1823. CrossRef Medline

Zimmermann E, Lappe M (2009) Mislocalization of flashed and stationary visual stimuli after adaptation of reactive and scanning saccades. J Neurosci 29:11055-11064. CrossRef Medline

Zimmermann E, Lappe M (2010) Motor signals in visual localization. J Vis 10:2. CrossRef Medline

Zimmermann E, Burr D, Morrone MC (2011) Spatiotopic visual maps revealed by saccadic adaptation in humans. Curr Biol 21:1380-1384. CrossRef Medline 